\title{
Seroconversion rates following 2 doses of Measles- Mumps- Rubella Vaccination Given at the Ages 12 and 18 Months: Data for possible additional dose at older age
}

\section{Hana Saffar}

IKHC. Teheran University of Medical Sciences

Sayed Jaber Mousavi

Mazandaran University of Medical Sciences

Hiva Saffar

Teheran University of Medical Sciences

Mohammad-Reza Parsaei

Mazandaran University of Medical Sciences

Gholam-Reza Ghorbani

Mazandaran University of Medical Sciences

Mohammad-Jafar Saffar ( $\sim$ Saffar@softhome.net)

Mazandaran University of Medical Sciences

\section{Research Article}

Keywords: Immunogenicity, MMR, Iran, Measles, Mumps, Rubella, Elimination, primary vaccine failure.

Posted Date: May 10th, 2021

DOl: https://doi.org/10.21203/rs.3.rs-460947/v1

License: (c) (i) This work is licensed under a Creative Commons Attribution 4.0 International License.

Read Full License

Version of Record: A version of this preprint was published at BMC Immunology on January 16th, 2022. See the published version at https://doi.org/10.1186/s12865-021-00465-1. 


\section{Abstract}

Background. Despite high rate of vaccination coverage with 2-doses of measles vaccine among Iranian children, outbreaks of measles occurred among different age groups and fully vaccinated subjects. Although the main reason for these outbreaks is unknown, however, vaccine failure was supposed to be an important cause. This study was designed to determine the immunogenicity of measles- mumpsrubella (MMR) vaccine currently in use among Iranian children.

Methods. This prospective study was conducted among ${ }^{3} 12$ month- old healthy children who were candidates of scheduled MMR vaccination. Blood samples were obtained from each mother- infant pair just before vaccination, and from infants 4- 6 weeks after $\mathrm{MMR}_{1}$ and $\mathrm{MMR}_{2}$ immunization. Collected sera were tested for specific IgG antibodies against MMR agents using ELISA method. The proportion of seroprotected subjects among mother- infant pairs before vaccination as well as the prevalence rates of seroconversion after $\mathrm{MMR}_{1}$ and $\mathrm{MMR}_{2}$ vaccination were calculated. Collected data were analyzed using descriptive statistical methods.

Results. During 22-months study period, 92 mother- infant pairs were participated. Seroimmunity rates against MMR viruses were $85.8 \%, 84.7 \%$ and $86.9 \%$ for mothers, and $3.2 \%, 2.1 \%$ and $1.0 \%$ for children, respectively. After $\mathrm{MMR}_{1}$ vaccination of 52 seronegative children, $80.7 \%, 78.8 \%$ and $75 \%$ were seroconverted. These rates increased to $94.8 \%, 89.7 \%$ and $92.3 \%$ after the $\mathrm{MMR}_{2}$ vaccination. Also, the specific immunity was enhanced among seropositive children.

Conclusion. Majority of the mothers and few infants were immune to MMR viruses, prior to $M M_{1}$ vaccination. Seroconversion rates detected after $\mathrm{MMR}_{1}$ injection, and overall seroprotection rates achieved after 2-doses of MMR vaccination were less than expected and inadequate to preserve longterm protection against MMR agents.

\section{Background}

Measles, mumps, and rubella (MMR) are communicable viral illnesses that are preventable through vaccination. Measles is a highly contagious infection that can be transmitted to more than $90 \%$ of susceptible subjects and is still a major cause of death among children, particularly in children less than 5 years old [1]. About 140000 measles deaths were reported worldwide in 2018 [2]. From first of Januaryto the end of July of 2019, more than 364806 cases of measles were reported from 182 countries in the world; this rate surpassed $23 \%$ from the similar period in 2018[2-3].

Mumps, an acute contagious disease, most often affects susceptible children to young adults in the closed crowded community and can be associated with serious complications such as:

meninigoencephalitis, orchitis, pancreatitis, myocarditis, and nephritis [4,5]. Rubella, a mild exanthematous infection, can be a real treat when infecting pregnant women especially in the first trimester and can result in fetal loss or devastating multiple anomalies known as congenital rubella 
syndrome (CRS) [6, 7]. According to world health organization (WHO) and other authorities, all children should receive a second dose of MMR vaccine to protect them against primary vaccine failure (PVF), and also to boost antibody titers of the primary responders in order to provide enhanced protection against secondary vaccine failure (SVF) [1, 8-11]. After implementation of 2 doses MMR vaccine scheduled after the age of 12 months with high vaccine coverage rate, the incidence of these infections reduced significantly and/ or eliminated in some WHO regions or countries [1, 8-11], however, during recent years, outbreaks of these infections particularly measles and mumps occurred, even in those subjects who were fully MMR vaccinated and in countries where endemic transmission was interrupted [12-16]. Decreasing seroimmunity prevalence rate and reduced vaccine efficacy overtime have been proposed as the main causes of these resurgence $[4,8,9,17-19]$.

While considering vaccine failure, it should be explained that either primary or secondary vaccine failure is desired [20].

Following universal 2 doses scheduled monovalent measles vaccine $(\mathrm{mMV})$ immunization of the Iranian children from 1984, and the national measles - rubella campaign of the 5-25 year- old population in December 2003, the incidence of measles cases in the Iran reduced markedly[21, 22]. However, despite the high vaccine coverage rates during recent years, small outbreaks of measles have occurred even among fully vaccinated subjects in some regions of the country $[15,16]$. The main reasons for these outbreaks are not clear.

Previous measles seroprevalence studies conducted in the Iran showed a gap between immunization coverage rates and the proportion of those who were seroprotected [23]. The extent of this gap is influenced by several factors including age at the time of initial immunization and time elapsed since the last vaccination, as well as vaccine related factors such as: type of the virus strain, and cold chain regulation in health care centers [24-27]. In order to determine the immunogenicity of MMR vaccine among Iranian children, some studies were performed. The seroconversion rates against MMR agents observed in these studies varied markedly. For example, in a study by Shamsizadeh, et al, from Ahwaz, six months after administering $\mathrm{MMR}_{1}$ to $>12$ month-old infants, seroconversion rates for measles, mumps and rubella were $42.9 \%, 58.6 \%$ and $90 \%$, respectively. These rates were $45.6 \%, 76.7 \%$ and $87.8 \%$, 6 months after given MMR vaccine to 6 year- old children who were vaccinated earlier with 2 doses of $\mathrm{mMV}$ [28]. Also, in another study, after administering the first dose of MMR vaccine to 12-15 month- old children, seroconversion rate for measles was lower than global reported rate; $75.8 \%, 95.3 \%$ and $73.8 \%$ for measles, mumps and rubella, respectively [29]. However, in a study among > 12 month- old children under the strict control of vaccination technique and cold-chain regulation by trained vaccinators, seroconversion rate following $\mathrm{MMR}_{1}$ was $91.2 \%$ for measles [30]. These findings raised concern about possible role of PVF as a cause of measles outbreaks occurred in fully vaccinated individuals, possibly because of poor control of cold chain and/or possible persistence of specific antibody beyond 12 months of age [28-31]. 
This study was designed to investigate the immunogenicity of the MMR vaccine currently in use among a cohort of more than 12 month-old children following 2 doses of MMR vaccine given at the ages of 12 and 18 months. Also, the seroprevalence rates of specific immunity to MMR agents among children just before their immunization and its possible effects on the vaccine immunogenicity were determined.

\section{Methods And Subjects}

Over a 22 months study period (1 October 2017 to 31 July 2019) healthy children aged $\geq 12$ month-old, who were brought to the primary health care centers for scheduled MMR immunization program in East of Mazandran province, North of Iran on a voluntary basis were recruited. Children with acute illness, those with history of chronic diseases, malignancies, immunodeficiency, febrile exanthematous diseases, recipients of blood/ blood products; additional MMR or any measles containing vaccines, history of prematurity (gestational age $<36$ weeks and/or birth weight $<2500$ grams), and any contraindications to MMR vaccination were excluded. Standard ethical guidelines and protocols were used and the study was approved by the Ethic Committee of the Mazandran University of Medical Sciences; IR.

MAZUM.REC1390.3073. After obtaining informed written consent from guardian, $3 \mathrm{cc}$ blood from each infant- mother pair was drawn. Four- to 6 weeks after vaccination with MMR vaccine $\left(M_{M} R_{1}\right)$ : [Domestic Razi institute of serum and vaccines: Karaj- Iran (Measles: AlK- C, Mumps: Hoshino, Rubella: Takahashi all CCID50 1000), or MMR vaccine: Serum Institute of India: (Measles: Edmonston- Zagreb; 1000 CCID 50, Mumps; Leningrad- Zagreb CCID50 5000; Rubella wistar RA27/5 CCID50: 1000)], 3 CC blood was obtained from vaccinated infants. Vaccines were dispensed in multi-dose (2, 5 dose/vial) and were stored at $2-8^{\circ \mathrm{C}}$ and reconstituted before vaccination. Reconstituted unused vaccines were discarded after 6 hours. Collected sera were stored at- $20^{\circ} \mathrm{C}$. Based on the national vaccination program in Iran, these children cameback for scheduled second dose of MMR vaccine $\left(\mathrm{MMR}_{2}\right)$ after 6 months. Four- to 6 weeks after $\mathrm{MMR}_{2}$, 3rd venous blood samples were obtained. All collected sera (mother and infant pairs) were tested for specific IgG against measles, mumps and rubella with ELISA methods, using qualitative Vircell microbiologist (measles IgG/ IgM G/M 1001, mumps IgG/ IgM, G/ M 1014, and Rubella Ig G. G/ 1026) kits, in the University laboratory. According to manufacturer's instructions, first, the mean OD for calibrator was measured and then cut- Off value (Kit Content) was calculated. The results were interpreted as antibody index which was calculated as: (sample OD/cut-off serum OD) $\times 10$. Samples with antibody indices $<9$ were considered as negative (not containing protective specific antibody), 9-11 equivocal and $>11$ as positive (seroprotected and immune). Equivocal samples were rechecked, and if $<11$, called as negative and $>11$ as positive. This categorization was applied for three MMR agents, ELISA Kit for rubella was standardized to $\mathrm{WHO}$ international $10 \mathrm{Ul} / \mathrm{mL}$. The prevalence rates of immunity against each agent among mother-infant pairs before vaccination were calculated. After $\mathrm{MMR}_{1}$, the proportion of responders and mean concentration of antibodies (MCA) for each agent were determined. After $\mathrm{MMR}_{2}$, the frequency of seroconversion rates among nonresponders to $\mathrm{MMR}_{1}$ and $\mathrm{MCA}$ levels of both new responders and seroimmune subjects were calculated. Collected data were analyzed using SPSS version of 16.0. The descriptive statistical method was used in the form of percentile for seroconversion and response rate, 
and Chi-Square and students T-test to find differences between variables as appropriate. Results were considered to be statistically significant when the $P$ value was less than 0.05 .

\section{Results}

For this study, during 22 months of study period, 92 mother- infant pairs were participated. 92 infants include 43 females and 49 males; the mean age of mothers was 27.4 years (age range 21-42 years) and the mean age of children for $\mathrm{MMR}_{1}$ and $\mathrm{MMR}_{2}$ vaccination was 12.1 and 18.3 months respectively. After the $\mathrm{MMR}_{1}$ vaccination, of 92 participated children, 52 (56.5\%) blood samples were obtained from children, whereas after $\mathrm{MMR}_{2}$ vaccination, 39 (42.4\%) participants agreed to give blood samples. As are presented in Table 1, approximately, $85.8 \%, 84.7 \%$ and $86.9 \%$ of mothers were serologically immune against $M-M-R$, agents, and 3, 2, 1 infants retained their seroprotection against these agents, Just before $M M R_{1}$ vaccination, respectively.

After administration of $\mathrm{MMR}_{1}$ vaccine, nearly $84.6 \%, 82.7 \%$ and $78.8 \%$ of seronegative vaccinated children responded to $M-M-R$ agents of vaccine and became lgG seroconverted. The MCAs for three MMR agents were $22.20 \pm 6.35,18.40 \pm 5.15$ and $21.30 \pm 5.76$, respectively. After the $\mathrm{MMR}_{2}$ injection among susceptible subjects, 4 out of $6(66.6 \%)$ responded to measles, 3 out of $7(42.8 \%)$ responded to mumps, and 8 of $10(80 \%)$ responded to rubella, and seroprotection rates for each virus approached to $94.8 \%$ for measles, $89.7 \%$ for mumps and $92.3 \%$ for rubella. The acquired MCAs were significantly higher than those after $\mathrm{MMR}_{1}$, indicating boosting of immunity. MCA was, $28.44 \pm 6.17 \mathrm{VS} 22.20 \pm 6.35 \mathrm{P}=$ 0.003 for measles and $26.67 \pm 5.80$ VS $18.40 \pm 5.15, P<0.001$ for mumps $27.08 \pm 7.68$ VS $21.30 \pm 5.76, P$ $=<0.001$ for rubella. Seroimmunological characteristics of mother-infant pairs and the patterns of immune response to administering $M M_{1}$ and $M M R_{2}$ vaccine for three viral strains are presented in Table 1. 
Table 1

Measles- Mumps- Rubella Seroprevalence profiles among mother-infant pairs just before MMR immunization, and seroconversion rates following administering 2-doses of MMR vaccine giving at the ages 12 and 18 months, Sari-Iran 2018.

\begin{tabular}{|c|c|c|c|}
\hline MMR agents & $\begin{array}{l}\text { Measles: } n(\%), \\
\text { MCA } \pm \text { SD }\end{array}$ & $\begin{array}{l}\text { Mumps: } n(\%) \\
\text { MCA } \pm \text { SD }\end{array}$ & $\begin{array}{l}\text { Rubella: } n(\%) \\
\text { MCA } \pm \text { SD }\end{array}$ \\
\hline Mothers $n=92$ & $\begin{array}{l}79(85.8 \%), 22.40 \\
\pm 7.25\end{array}$ & $\begin{array}{l}78(84.7 \%), 21.30 \\
\pm 5.76\end{array}$ & $\begin{array}{l}80(86.9 \%) 19.18 \\
\pm 4.32\end{array}$ \\
\hline Infants $n=92$ & $3(3.2 \%)$ & $2(2.1 \%)$ & $1(1.0 \%)$ \\
\hline MMR1 n = 52 & $\begin{array}{l}44(84.6 \%), 22.20 \\
\pm 6.35\end{array}$ & $\begin{array}{l}43(82.7 \%), 18.40 \\
\pm 5.15\end{array}$ & $\begin{array}{l}41(78.8 \%), 21.30 \\
\pm 5.76\end{array}$ \\
\hline MMR2 n = 39 & $\begin{array}{l}37(94.8 \%), 28.44 \\
\pm 6.17\end{array}$ & $\begin{array}{l}35(89.7 \%), 26.67 \\
\pm 5.80\end{array}$ & $\begin{array}{l}36(92.3 \%), 27.08 \\
\pm 7.68\end{array}$ \\
\hline $\begin{array}{l}\mathrm{P} \text { value between } \mathrm{MCA} \text { after dose } \\
\mathrm{MMR}_{1} \text { and } \mathrm{MMR}_{2}\end{array}$ & $P=0.003$ & $P<0.001$ & $P<0.001$ \\
\hline
\end{tabular}

\section{Discussion}

In this study, nearly $85 \%$ of investigated mothers were serologically immune to MMR viruses. Also, few more than 12 months' infants showed seroprotection against these agents before their scheduled $\mathrm{MMR}_{1}$ vaccination. After administering the $\mathrm{MMR}_{1}$ to more than 12 month- old children, nearly $84,6 \%, 82,7 \%$ and $78.8 \%$ of vaccinated children responded to three components of MMR1 vaccine, respectively and seroconverted. Six months after the initial MMR immunization, most serosusceptible children showed immunologic response to $\mathrm{MMR}_{2}$ and serologicaly achieved protection to MMR agents. Also, their earlier acquired seroimmunity following first dose of MMR vaccine injection was boosted. Finally, following administering 2- doses of MMR vaccine to children after the age of 12 months, approximately $94.8 \%$, $89.7 \%$ and $92.3 \%$ of vaccinees acquired seroprotection to measles, mumps, and rubella, respectively.

Since primary protection against infectious diseases is provided mainly by maternal $\lg$ antibodies transferred actively via placenta to the fetus, higher maternal antibodies are associated with increased levels of antibodies in the offspring. However, these antibodies disappear during first few months of life. Higher levels will persist for a longer time. Giving booster doses of vaccine or natural exposure to these agents among childbearing age women, can result in robust of their specific immunity and consequently higher levels of passive immunity will appear in the infants. Therefore, the concentrations of specific antibodies, particularly anti-measles in the infants may vary widely in the world [32,33].

Based on this study findings, most participated mothers were seropositive against three agents of vaccine. In addition to history of measles immunization during childhood, majority of our mothers were reimmunized in the national program of MR campaign conducted at the December 2003 [21]. However, 
none of these mothers were immunized against mumps earlier and their immunity against mumps is the results of natural infection. Level of antibody achieved by natural infections is usually higher and it persists for a longer time compare to vaccine - induced immunity [32]. Therefore, this may explain the high rates of immunity against mumps observed in our studied mothers. The high rates of immunity against measles and rubella may be due to positive impact of MR reimmunization performed 13to15years earlier or it could be the result of exposure to natural infection in the past. Similar results are reported recently among Iranian childbearing age women with the same age [34]. In a nationwide seroprevalence study among Iranian girl at the verge of marriage that were MR reimmunized 13-14years earlier, results showed that $80,7 \%$ (70.3-to $89.5 \%)$ and $90.6 \%$ (81.2-to 95\%) were immune to measles and rubella, respectively. There was a sharp difference between those who were MR vaccinated and those who were not. They concluded that these high rates of seroimmunity were the positive impact of MR revaccination [34].

According to our data, a few children were seroimmune to MMR agents just before $\mathrm{MMR}_{1}$ vaccination detected by ELISA method. The exact origin of these antibodies is not clear, but most probably is maternal. In a similar study from the region among 112 mother-infant pairs in year 2008, (5-years after the national MR campaign), nearly $6.2 \%$ and $10.7 \%$ of 12 month- old infants, all from MR reimunized mothers, retained their seroimmunity to measles and rubella, respectively [35]. Also, in another similar study from southeast of Iran, nearly $3.7 \%$ of 12 months old infants just before $\mathrm{MMR}_{1}$ vaccination were serologically immune to measles detected by ELISA[30]. The point of concern is that ELISA methods is not sensitive enough to detect low titers of measles antibodies. Therefore, if more sensitive methods are used, higher proportion of infants may retain their passive immunity just before $\mathrm{MMR}_{1}$ immunization [36]. MMR vaccine is a live attenuated vaccine and presence of specific antibodies could negatively impact on the immunogenicity of the person. After giving scheduled $\mathrm{MMR}_{1}$ vaccine to more than 12 month- old seronegative children in this study, nearly $15 \%, 17 \%$, and $21 \%$, of vaccinated children remained seronegative to MMR agents, respectively [primary vaccine failure (PVF)]. Failure to seroconversion (PVF) is possibly due to the persistence of maternal antibodies in children vaccinated at younger age and is believed to be the principal reason to why some vaccinees remained susceptible to measles [20], although vaccine-related factors such as inappropriate handling of the vaccine and poor cold-chain regulation should not be ignored $[26,28,30,37]$. In this regard, in our earlier study the impact of specific antibodies on the immunogenicity of MMR vaccine giving after the age of 12 months was investigated. Results showed that lower serocoversion rates and lower levels of specific antibodies were observed among infants with retained maternal antibodies beyond the age of 12 months than those without retained maternal antibodies [35]. Also, in a comparative study between two groups of children that were vaccinated with 2 doses of MMR vaccine scheduled at the ages of 15 months and 6 years VS 12 and 18 months, the immunogenicity of MMR vaccine was investigated. Results showed lower rates of seroconversion among both groups of children just before giving MMR2: 74\%, 82.3\%, and 68\% VS 78.9\%, $68 \%$ and $68 \%$ respectively. After $\mathrm{MMR}_{2}$, these rates approached to $94.4 \%, 94.4 \%$ and $92.4 \%$ VS $98.2 \%$, $97 \%$, and $87 \%$, respectively [38]. Similar to our results were also reported in one study from Tehran [29]. In that study which was performed by Tabatebaei et al, the immunogenicity of $\mathrm{MMR}_{1}$ vaccine was 
investigated among 12-15 month-old Tehranian children. Their results showed that following $\mathrm{MMR}_{1}$ vaccination, $75,8 \%, 95,3 \%$, and $73,8 \%$, of vaccinated children were seroconverted, to measles, mumps, and rubella respectively [29]. Furthermore, in a similar study, the immunogenicity of MMR vaccine administered to $\geq 12$ month-old children under the strict control of researchers for cold-chain regulation, the seroconversion rate was $91.2 \%$ [30]. The possible negative impact of poor cold-chain controlling on the immunogenicity of measles containing vaccine was also postulated by other researchers [28, 37]. When comparison was made between our findings and other data from the Iran with those that were reported worldwide [1, 8, 11, 24, 25], the rates of PVF detected in Iranian studies were higher. In most of these studies administering $M_{M R}$ vaccine at the age 12-13 months, was associated with $90-95 \%$ seroconversion rates for M-R component of the MMR vaccine $[1,24,25]$. The main possible reason for the higher rates of PVF observed in our study as well as other studies from Iran may be due to presence of low concentrations of specific antibodies, particularly anti- measles antibody, undetectable by ELISA method, and its negative influence on the immunogenicity of the MMR vaccine. However, the possible negative impact of technical problems in vaccination procedure should be also considered. Further studies are recommended to investigate the presence of specific antibodies, particularly measles antibody by a more sensitive method just before $\mathrm{MMR}_{1}$ immunization at the age 12-13months as well as its effects on the immunogenicity of MMR vaccine. Also, periodic educational sessions for vaccinators are suggested to improve vaccination techniqes, particularly cold-chain regulation.

Most serosusceptible children following $\mathrm{MMR}_{1}$, acceptably seroconverted with boosted antibodies after administration of $\mathrm{MMR}_{2}$ at the age of 18 months. However the overall seroprotection rates detected in this study were lower than expected in the world. Results of most studies indicated that vaccination with 2-doses of MMR vaccine after the age of 12 months is associated with $>95-98 \%$ seroconversion rate [1, $8-11]$, but the results of most studies from Iran vary considerably $[28,38]$. While the result of one study from north of Iran showed seroconversion rates of $98,2 \%$ and $94,4 \%$ after $M_{M M R}$ given at the age of 6 years or 18 months [38], respectively, in another study from Ahwaz 6 months after MMR vaccination of 6.5 year- old children with history of 2-doses of monovalent measles vaccination at the ages of 9 and 15 months, the seroprevalence rate was $45.6 \%$, for measles, $76.7 \%$ for mumps and $87.7 \%$ for rubella [28]. However, our data in this study indicated acceptable, but not optimal seroprotection rates among Iranian children following two-doses of MMR immunization.

While considering these rates of seroprotection along with $97 \%$ of vaccine coverage rates in all districts of the country a population immunity rate of $89.9 \%, 87.6 \%$ and $89.9 \%$ for MMR agents could be estimated, respectively. The concerning point is that vaccine-induced antibodies against measles and mumps decrease faster over time compare to rubella [17-19] (secondary vaccine failure). Therefore, an increasing number of potentially measles-mumps susceptible population will accumulate in the community and facilitate outbreaks even among fully vaccinated subjects [12-14]. However, this rates of herd immunity against measles is lower than is required to eliminate/sustain measles elimination [ 811]. The rates for mumps and rubella are at the lowest threshold to eliminate mumps and rubella epidemics $[4,8]$. Therefore, in mid-and long-term, these levels of immunogenicity are challenging, and 
raise concern about the sustaining measles- rubella elimination in the country with certificate of elimination which was achieved in the last 2 years [39]. In this regard, monitoring and controlling coldchain regulation in the primary health care centers to preserve vaccine potency, and periodic serosurveillance to monitor immunity against MMR agents are recommended. Further studies with larger sample size using more sensitive laboratory methods to measure low levels of specific antibodies just before MMR1 immunization are suggested. If these results are confirmed by further studies, changing the age of the first dose of MMR vaccine to 14 to15 months and/or considering additional universal dose of MMR vaccine at the older age are recommended.

The main limitations of this study include its small sample size and also using two brands of MMR vaccine interchangeably that may influence the final results.

\section{Conclusions}

Based on the study findings, the immunogenicity of the MMR vaccine currently in use in the country is acceptable in short-term. However, to maintain mid-to long-term herd immunity, national or regional supplementary immunization activities seem reasonable. Similar studies with larger sample size in different regions of the country are recommended to measure specific antibody concentration particularly measles antibody with a more sensitive method before first dose of MMR administration and also consider strict control of cold-chain and vaccine administering techniques to assess the MMR vaccine immunogenicity.

\section{Abbreviations}

MMR: Measles Mumps Rubella, ELISA: enzyme immune assay, CRS: congenital rubella syndrome, WHO: world health organization, PVF: Primary Vaccine Failure, mMV: monovalent measles Vaccine, MAZUMS: Mazandaran University of Medical Sciences, IgG: immunoglobulin G, MCA: mean Concentration Antibodies, MR: measles-rubella.

\section{Declarations}

Ethic approval and Consent to Participate: The study was provided ethical approval by the MAZUMS No: IR, MAZUMS, Rec. 3082. The study obtained the consent of all participants and signed and informed consent form prior to investigation. They were assured about confidentially and that their contribution would be on a voluntary basis as well as that they had full rights to withdraw from the study at any time.

Consent for Publication: Not Applicable.

Availability of data and materials: The datasets used and/or analyzed during the current study are available from the corresponding author on reasonable request

Competing interests: The authors declare that they have no competing interest 
Funding: This study was funded by Vice-chancellor for research and Technology MAZUMS No: (IR, MAZUMS, Rec. 3082). The funder had no role in the design of the study and collection, analysis, interpretation of collected data and writing of manuscript.

Authors Contribution and Details: Hn.S Involved in study design, literature search, laboratory testing and writing the paper. J.M. data collection and statistical analysis and interpretation. Hv.S. Involved in study design, literature search, laboratory testing and writing the paper. M.P. and G.G. involved in recruiting, interview and blood sampling. M.S. all phases of the study.

Acknowledgment: The researchers would like to thank, the students for their participation in this study, as well as the staff in each faculty. Also, we appreciate the vice-chancellor for cultures and students for their assistance and recruiting students to participate in the study. Finally, special thanks to Nakhaei SM for his help in interviewing and blood sampling.

\section{References}

1. World Health Organization. Measle. WHO position paper. WKLY Epidemiol Rec.17. 2017:92:205-228.

2. World Health Organization. Measle. Key facts:5 Dec 2019. www.WHO.int/news-room/factsheet/detail measles.

3. Center for Disease Control and Prevention (CDC). Global Health. Global measles outbreaks. cdc.gov/global Health/measles /global measles outbreaks.html.

4. Lam E, Basen JB, Zucker JR. Mumps: An update on outbreaks, vaccine efficacy and Genomic diversity. Cli Microbiol Rev. 2020:33(2).

5. Center for Disease Control and Prevention (CDC). Mumps for Health-care providers. www.cdc.gov/mumps/hep.html.

6. Lambert N, Strebel P, Orenstein W, Icenogle J, Poland GA. Rubella. Lancet. 2015;385(9984):2297-307.

7. World Health Organization. Rubella. Key facts: 4 Oct 2019. www.WHO.int/news-room/factsheets/detail/Rubella.

8. Bankamp B, Hickman C, Icenogle JP, Rota PA.Successes and challenges for preventing measles, mumps and rubella by vaccination. Curr Opin Virol. 2019 Feb;34:110-116.

9. Holzmann $\mathrm{H}$, Hengel $\mathrm{H}$, Tenbusch M, Doerr HW. Eradication of measles: remaining challenges. Med Microbiol Immunol. 2016;205(3):201-8.

10. Orenstein WA, Cairns L, Hinman A, Nkowane B, Olivé JM, et al. Measles and Rubella Global Strategic Plan 2012-2020 midterm review report: Background and summary. Vaccine. 2018;36 Suppl 1:A35A42.

11. Hinman AR. Measles and rubella eradication. Vaccine. 2018;36(1):1-3.

12. Defay F, De Serres G, Skowronski DM, Boulianne N, Ouakki M, et al. Measles in children vaccinated with 2 doses of MMR. Pediatrics. 2013;132(5):e1126-33. 
13. Eom H, Park Y, Kim J, Yang JS, Kang H, et al. Occurrence of measles in a country with elimination status: Amplifying measles infection in hospitalized children due to imported virus. PLoS One. 2018;13(2):e0188957.

14. De Serres G, Markowski F, Toth E, Landry M, Auger D, et al. Largest measles epidemic in North America in a decade-Quebec, Canada, 2011: contribution of susceptibility, serendipity, and superspreading events. J Infect Dis. 2013:15;207(6):990-8.

15. Piri N, Karami M, Tapak L, Zahraei SM, Mohammadi Y. Monitoring progress towards the elimination of measles in Iran: supporting evidence from 2014 to 2016 by application of measles outbreaks data. BMC Public Health.2019;19(1):687.

16. Karami M, Zahraei SM, Sabouri A, Soltanshahi R, Biderafsh A, et al. Documentation of Measles Elimination in Iran: Evidences from 2012 to 2014. J Res Health Sci. 2017;17(3):e00387.

17. Smetana J, Chlibek R, Hanovcova I, Sosovickova R, Smetanova L, et al. Decreasing Seroprevalence of Measles Antibodies after Vaccination - Possible Gap in Measles Protection in Adults in the Czech Republic. PLoS One. 2017;12(1):e0170257.

18. Kang HJ, Han YW, Kim SJ, Kim YJ, Kim AR, et al. An increasing, potentially measles-susceptible population over time after vaccination in Korea. Vaccine. 2017;35(33):4126-4132.

19. Kontio M, Jokinen S, Paunio M, Peltola H, Davidkin I. Waning antibody levels and avidity: implications for MMR vaccine-induced protection. J Infect Dis. 2012;206(10):1542-8.

20. Pebody RG, Gay NJ, Hesketh LM, Vyse A, Morgan-Capner P, et al. Immunogenicity of second dose measles-mumps-rubella (MMR) vaccine and implications for serosurveillance. Vaccine. 2002;20(78):1134-40.

21. Zahraei SM, Gouya MM, Azad TM, Soltanshahi R, Sabouri A, et al. Successful control and impending elimination of measles in the Islamic Republic of Iran. J Infect Dis. 2011;204 Suppl 1:S305-11.

22. Teleb N, Lebo E, Ahmed H, Hossam AR, El Sayed el T, et al. Progress toward measles elimination Eastern Mediterranean Region, 2008-2012. MMWR Morb Mortal Wkly Rep. 2014 Jun 13;63(23):5115.-

23. Izaidi S, Mokhtari-Azad T, Zahraei S.M. Measles vaccination coverage and seroprevalence of antimeasles antibody in south-east Islamic Republic of Iran. Eastern Mediterranean Health Journal. 2015;21(6): 396-402.

24. Perez SC, De Serres G, Bureau A, Skowronski DM Reduced Antibody Response to Infant Measles Vaccination: Effects Based on Type and Timing of the First Vaccine Dose Persist After the Second Dose. Clin Infeci Dis 2017;65(7):1094-1102.

25. Redd SC, King GE, Heath JL, Forghani B, Bellini WJ, et al. Comparison of vaccination with measlesmumps-rubella vaccine at 9, 12, and 15 months of age. J Infect Dis. 2004;189 Suppl 1:S116-22.

26. Saffar H, Saffar MJ, Saffar H. Vaccination in developing countries: a review of probable factors for lower responses to vaccines. J of Pediatr Rev, 2013;1(1):12-18.

27. Carazo S, Billard MN, Boutin A, De Serres G. Effect of age at vaccination on the measles vaccine effectiveness and immunogenicity: systematic review and meta-analysis. BMC Infect Dis. 
2020;20(1):251.

28. Shamsizadeh A, Nikfar R, Makvandi M, Hakimzadeh M, Alisamir, et al. Seroprevalence of measles, mumps and rubella Antibodies in 18 months and 6.5 years old children: 6 months after measlesmumps-rubella (MMR) vaccination. Jundishapur J Microbiol. 2012;5(4):578-581.

29. Rafiei Tabatabaei S, Esteghamati AR, Shiva F, Fallah F, Radmanesh R, et al. Detection of serum antibodies against measles, mumps and rubella after primary measles, mumps and rubella (MMR) vaccination in children. Arch Iran Med. 2013;16(1):38-41.

30. Zahraei SM, Izadi S, Mokhtari-Azad T. Factors affecting the seroconversion rate of 12-month-old babies after the first injection of measles vaccine in the southeast of Iran. Hum Vaccin Immunother. 2016;12(12):3118-3124.

31. Izadi S, Zahraie SM, Sartipi M. An investigation into a measles outbreak in southeast Iran. Jpn J Infect Dis. 2012;65(1):45-51.

32. Leuridan E, Hens N, Hutse V, leven M, Aerts M, et al. Early Waning of Maternal Measles Antibodies in Era of Measles Elimination: Longitudinal Study. BMJ. 2010;340:c1626.

33. Leuridan E, Van Damme P. Passive transmission and persistence of naturally acquired or vaccineinduced maternal antibodies against measles in newborns. Vaccine. 2007;25(34):6296-304.

34. Zahraei SM, Mokhtari-Azad T, Izadi Sh, Mohammadi M, Sabouri A. Seroprevalence of anti-rubella and anti-measles antibodies in women at the verge of marriage in Iran. Vaccine. 2020 Jan 10;38(2):235241.

35. Saffar MJ, Ajami A, Khalilian AR, Saffar H. The impact of maternal measles-rubella immunization on the 12-month-old infant's immune response to measles-mumps-rubella vaccine immunogenicity. Eur J Clin Microbiol Infect Dis. 2009;28(7):845-7.

36. Ratnam S1, Gadag V, West R, Burris J, Oates E, et al. Comparison of commercial enzyme immunoassay kits with plaque reduction neutralization test for detection of measles virus antibody. J Clin Microbiol. 1995;33(4):811-5.

37. Moghadam M, Afsarkazerooni P, Ebrahimi M, Soltani M, Razmpoor A, et al. Measles outbreak in South of iran, where vaccine coverage was high: a case-series study. Iran J Public Health. 2014;43(3):375-80.

38. Saffar MJ, Fathpour GR, Parsaei MR, Ajami A, Khalilian AR, et al. Measles-mumps-rubella revaccination; 18 months vs. 4-6 years of age: potential impacts of schedule changes. J Trop Pediatr. 2011;57(5):347-51.

39. Teleb N, Atta H, Hajjeh R. Measles and rubella elimination in the Eastern Mediterranean Region: successes and challenges. East Mediterr Health J. 2019;25(10):667-8. 